\title{
Polyamines in human breast milk for preterm and term infants
}

\author{
J. Plaza-Zamora ${ }^{1} \dagger$, M. Sabater-Molina ${ }^{1} \dagger$, M. Rodríguez-Palmero ${ }^{2}$, M. Rivero ${ }^{2}$, V. Bosch ${ }^{3}, J$ M. Nadal ${ }^{3}$, \\ S. Zamora ${ }^{1}$ and E. Larqué ${ }^{1 *}$ \\ ${ }^{1}$ Department of Physiology, Faculty of Biology, Campus Mare Nostrum, University of Murcia, 30100, Murcia, Spain \\ ${ }^{2}$ Ordesa Group, Barcelona Scientific Park, Barcelona, Spain \\ ${ }^{3}$ Neonatology Service, Virgen de la Arrixaca Hospital, Murcia, Spain
}

(Submitted 25 May 2012 - Final revision received 17 October 2012 - Accepted 29 October 2012 - First published online 3 January 2013)

\begin{abstract}
Maternal milk is the first source of exogenous polyamines for the newborn. Polyamines modulate gut maturation in neonates, but no studies are available on polyamine concentration in human milk of preterm babies, even though they could be important for their immature gut. The present study aimed to determine polyamine concentration in human breast milk of mothers with preterm or term infants during the first month of lactation. Human milk samples were obtained during the first month of lactation from twenty-seven mothers with preterm babies and twelve mothers with babies born at term. The polyamine concentration in human milk was quantified by HPLC. During the first month of lactation, the total polyamine concentration was significantly higher in preterm milk than in term milk samples (7590 (SD 4990) v. 4660 (SD 4830) nmol/l, respectively $(P=0.034)$ ), as well as individual polyamine concentrations. Polyamine concentration in mature milk for preterm babies was significantly higher than that in mature milk for babies at term, and a similar trend was observed in colostrum and transition human milk. The spermidine/spermine ratio was higher in transition milk in preterm $v$. term samples, while in mature milk, the ratio was significantly lower in preterm than in term babies. In conclusion, the polyamine concentration was significantly higher in human milk for preterm than for term infants. This and the different spermidine/spermine ratios could influence the gut development of premature babies.
\end{abstract}

Key words: Polyamines: Spermidine/spermine ratio: Preterm: Human milk: Spermidine: Spermine

The polyamines putrescine, spermidine and spermine are polycationic substances of low molecular weight synthesised in all mammalian cells from their immediate precursor, ornithine. Polyamine metabolism includes intracellular de novo synthesis in a strictly controlled balance by several enzymes, the main one being ornithine decarboxylase ${ }^{(1-4)}$ The exogenous supply includes both polyamine intake from dietary foods as well as their synthesis by gut microbiota. The highest requirement for these aliphatic amines occurs in rapidly growing tissues, such as the mucosa of the gastrointestinal tract of newborns ${ }^{(5,6)}$. We have previously reported that milk formulas for newborn piglets supplemented with polyamines in concentrations resembling those of sow milk significantly enhanced crypt depth in the gut compared with control formulas with no added polyamines ${ }^{(7)}$, a finding that highlights the relevance of these compounds for gut maturation in early life.

Maternal milk is the first source of exogenous polyamines for newborn babies and animals ${ }^{(8-10)}$. Human milk contains relatively high levels of polyamines, mainly spermine and spermidine, with lower amount of putrescine ${ }^{(11)}$; their concentration in human milk increases during the first week postpartum, reaching a maximum after 1-2 weeks and then decreasing ${ }^{(10,12,13)}$. The polyamine concentration in human milk for term babies varies greatly among different studies $^{(9,10,12,14,15)}$, which makes it difficult to decide on a potentially suitable polyamine concentration for addition to infant formulas. The polyamine concentration in human milk depends on many factors, such as the time of breast milk collection, stage of lactation, the breast chosen, maternal polyamine dietary intake and maternal atopy, which also differs among countries ${ }^{(8,13,14,16,17)}$. In addition, the polyamine concentration in human milk of mothers with preterm babies is unknown, even though it is known that this type of milk differs in the concentration of key nutrients compared with term milk. The polyamine concentration in human milk for preterm babies could play a major role in their immature gut system, highlighting the relevance of increasing our knowledge on this biological fluid.

*Corresponding author: Elvira Larqué, fax +34 868 883963, email elvirada@um.es

† These authors contributed equally to this work. 
The aim of the present study was to evaluate whether the polyamine composition of human milk differs in mothers of preterm and full-term infants. For this, we evaluated the polyamine concentration in human breast milk for both preterm and term babies during the first month of lactation in a Mediterranean area, comparing the concentration in colostrum, transition milk and mature milk in both types of breast milk.

\section{Experimental methods}

Human milk samples were collected from two different groups of mothers delivering at the Universitary Hospital Virgen de la Arrixaca (Murcia, Spain). Of these, twelve women delivered at term (between weeks 37 and 42 of gestation) and twenty-seven women delivered prematurely (between weeks 28 and 36 of gestation). The present study was conducted according to the guidelines laid down in the Declaration of Helsinki, and all procedures involving human subjects/patients were approved by the Ethical Committee of the Virgen de la Arrixaca Hospital. Verbal informed consent was obtained from all subjects/patients. Verbal consent was witnessed and formally recorded.

Volunteers supplied samples of milk during the first month of lactation. The milk samples were collected from colostrum (4 (SD 1) d; 1-5d of lactation), transition (9 (SD 3) d; 6-14d of lactation) and mature milk (19 (SD 4) d; 15-30d) and later on frozen at $-80^{\circ} \mathrm{C}$ until polyamine analysis. For mothers at term, five samples of colostrum belonging to five different mothers, nine samples of transition milk from seven mothers, and five samples of mature milk from five mothers were obtained. Concerning mothers with preterm babies, five samples of colostrum belonging to five mothers, nineteen samples of transition milk from fifteen mothers, and twenty samples of mature milk from fourteen mothers were obtained. Only five mothers from the preterm group and five mothers from the term groups provided us with milk samples at the three different periods of lactation.

Human milk samples were collected by a manual pump and were promptly kept at $4^{\circ} \mathrm{C}$ as separate samples, and they were collected every day in the morning. Maternal age did not differ between mothers who delivered babies born preterm (aged 31.5 (SD 6.9) years; range from 17 to 44 years old) or at term (aged 30 (SD 5) years; range from 26 to 38 years old). Nevertheless, as expected, both gestational age (32.4 (SD 1.4) (ranged from 28 to 36) weeks $v .39 \cdot 5$ (SD 1.4) (ranged from 37 to 42) weeks) and birth weight (1745 (SD 359) v. 3543 (SD 514) g) were significantly lower in preterm than in full-term infants. Polyamines in human maternal milk were analysed using the AccQ-Fluor Reagent Kit (Waters S.A.), which uses 6-aminoquinolyl- $N$-hydroxysuccinimidyl carbamate as the derivatising reagent. 5-Sulphosalicylic solution $(0.2 \mathrm{~g} / \mathrm{ml}$; $200 \mu \mathrm{l}$ ) and $50 \mu \mathrm{l}$ of 1,6-diaminohexane (internal standard) were successively added to $1 \mathrm{ml}$ milk samples and then kept frozen overnight at $-80^{\circ} \mathrm{C}$. After centrifugation at $6700 \mathrm{~g}$ for $20 \mathrm{~min}$, the supernatant was recovered and centrifuged again at $6700 \mathrm{~g}$ for $10 \mathrm{~min}$; the supernatant was filtered through a $0.45 \mu \mathrm{m}$ Millipore filter prior to derivatisation. Borate buffer $(\mathrm{pH} 8.8 ; 70 \mu \mathrm{l})$ and $20 \mu \mathrm{l}$ AccQ Fluor (Waters) were added to
$100 \mu \mathrm{l}$ of the polyamine-containing sample for derivatisation and the mixture was incubated for $10 \mathrm{~min}$ at $55^{\circ} \mathrm{C}$. Derivatised polyamines were quantified by HPLC using a reverse-phase column (Nova-Pak C18, Waters S.A.). We used a gradient, starting with $25 \%$ phase A ( 20 mm-sodium acetate) and $75 \%$ phase $\mathrm{B}$ (methanol), reaching $100 \%$ of phase A after 30 min of running. Polyamine standards (putrescine dihydrochloride, spermidine trihydrochloride and spermine tetrahydrochloride) were purchased from Sigma Chemical Company.

\section{Statistical analyses}

The results were expressed as mean and standard deviation. When more than three samples of milk were collected from the same mother in the same stage of lactation, the mean value was calculated. The data followed a normal distribution evaluated by the Kolmogorov-Smirnov test. Comparisons between preterm and term human milk samples were analysed by $t$ test. Since some of the samples at each lactation period belong to different subjects, an ANOVA test followed by post hoc Bonferroni test was performed to analyse differences in polyamines among colostrum, transition milk and mature milk. The CV was calculated as $(\mathrm{sD} /$ mean $) \times 100$. All statistical analyses were carried out using SPSS for Windows (release 15.0; SPSS, Inc.). Differences were considered significant at $P<0.05$.

\section{Results}

In the present study, we found very low concentrations of putrescine compared with spermidine or spermine in human milk for term babies, a profile that was repeated in human milk for preterm babies (Table 1). Nevertheless, the concentration of individual polyamines (putrescine, spermidine and spermine) or total polyamines did not differ significantly between colostrum $(1-5 \mathrm{~d})$, transition milk (6-14d) and mature milk $(>15 \mathrm{~d})$, not only in term, but also in preterm milk samples (Table 1). This could be explained by the naturally high variability in the polyamine content of human milk samples, exceeding the $50 \% \mathrm{CV}$ at each lactation period when samples from different subjects are included among the lactation periods.

Polyamine concentration in mature milk for preterm babies was significantly higher than in mature milk for babies at term $(P<0 \cdot 01)$, and a similar change was also observed in colostrum and transition human milk, although the differences were not significant (Table 1). In fact, when all the samples collected during the first month of lactation were analysed together, human preterm milk showed a significantly higher polyamine concentration than the milk for babies born at term, as regards total polyamines, putrescine and spermidine, but only a trend in the case of spermine $(P=0.092)$ (Fig. 1). In addition, the spermidine/spermine ratio was significantly higher in transition milk for term babies, while in mature milk, this ratio was inverted, and was even below 1 in term infants (Table 1). 
Table 1. Polyamine concentration $(\mathrm{nmol} / \mathrm{l})$ in human milk of mothers with preterm and term infants at different stages of lactation (Mean values and standard deviations)

\begin{tabular}{|c|c|c|c|c|c|c|c|c|c|c|}
\hline & \multicolumn{2}{|c|}{ Putrescine } & \multicolumn{2}{|c|}{ Spermidine } & \multicolumn{2}{|c|}{ Spermine } & \multicolumn{2}{|c|}{$\begin{array}{c}\text { Total } \\
\text { polyamines }\end{array}$} & \multicolumn{2}{|c|}{$\begin{array}{c}\text { Spermidine/ } \\
\text { spermine ratio }\end{array}$} \\
\hline & Mean & $\mathrm{SD}$ & Mean & SD & Mean & SD & Mean & SD & Mean & $\overline{S D}$ \\
\hline \multicolumn{11}{|l|}{ Preterm } \\
\hline Colostrum $(n 5)$ & 59 & 69 & 279 & 241 & 351 & 422 & 689 & 609 & $1 \cdot 2$ & $1 \cdot 32$ \\
\hline Transition ( $n$ 15) & 36 & 36 & 339 & 225 & 340 & 230 & 715 & 434 & 1.0 & 0.36 \\
\hline Mature $(n 14)$ & 58 & 48 & 462 & 319 & 302 & 216 & 821 & 542 & 1.5 & 0.51 \\
\hline \multicolumn{11}{|l|}{ Term } \\
\hline Colostrum (n 5) & 14 & 31 & 178 & 158 & 279 & 321 & 472 & 465 & $1 \cdot 0$ & 0.50 \\
\hline Transition $(n 7)$ & 28 & 35 & 320 & 353 & 232 & 247 & 579 & 608 & $1 \cdot 4^{*}$ & 0.32 \\
\hline Mature $(n 5)$ & $29^{*}$ & 16 & $124^{\star}$ & 131 & $104^{*}$ & 77 & $257^{\star}$ & 108 & $0.6^{*}$ & 0.50 \\
\hline
\end{tabular}

${ }^{*}$ Mean values were significantly different from preterm milk samples $(t$ test) at each stage of lactation $(P<0.05)$.

\section{Discussion}

To date, there is no published study about polyamine concentration in human milk for preterm babies. In the present study, we reported that during the first month of lactation, the polyamine concentration in human milk for preterm babies was significantly higher than in breast milk for babies at term, which could influence gut maturation of premature infants and points towards a higher polyamine requirement in early life for preterm infants than for babies born at term. In addition, we found a significant change in the spermidine/ spermine ratio between preterm and term transition and mature human milk samples, which could be of interest for the addition of polyamines to infant formulas.

The polyamine concentration in human milk samples was highly variable; this would imply that a higher number of human milk samples should be collected to ascertain differences in polyamine concentration during the three stages of lactation. The polyamine concentration in human milk for infants at term varies greatly among studies, making it difficult to reach conclusions on the polyamine levels in human milk. The total polyamine concentration in our term milk samples were in the range of those reported by Buts et al. ${ }^{(14)}$ and Pollack et al. ${ }^{(9)}$. These authors reported a total polyamine concentration ranging between 1450 and $8600 \mathrm{nmol} / 1$ in the USA,

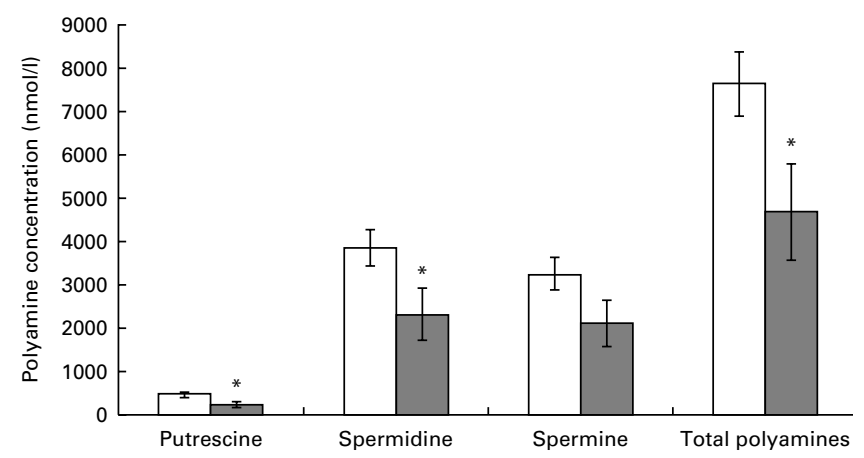

Fig. 1. Polyamine concentration in milk of mothers with preterm ( $\square$ ) and term ( $\square$ ) infants during the first month of lactation. Values are means, with standard deviations represented by vertical bars. * Mean value was significantly different from that for preterm milk. ( $P<0.05$; Student's $t$ test). while Romain et al. ${ }^{(10)}$ reported more than $10000 \mathrm{nmol} / \mathrm{l}$ in Belgium $(15030 \mathrm{nmol} / \mathrm{l})$, which is difficult to understand. Also, in Belgium, Buts et al. ${ }^{(14)}$ reported a total polyamine content in human breast milk of 5570 (SD 180) nmol/1, very different from that reported by Romain et al. ${ }^{(10)}$ and more similar to that mentioned by Pollack et $a l .{ }^{(9)}$. During the first week postpartum (colostrum samples), the present results are also in agreement with those provided by both Buts et al. ${ }^{(14)}$ and Pollack et al. ${ }^{(9)}$.

No significant differences in the concentration of total and individual polyamines were detected between different stages of lactation in term milk during the first month of lactation, perhaps because of the low number of samples collected. Some authors found that the concentration of polyamines in colostrum was much greater than that in mature breast milk $^{(10,14)}$. Pollack et al. ${ }^{(9)}$ reported that the polyamine concentrations in term milk samples during the first 1.5 months of lactation did not change, but concentrations of all three polyamines began to decrease after the first month and then fell sharply after the second month of lactation. The present results showed the highest values of polyamines in term milk samples at the end of the second week of lactation (total polyamine concentrations were 4810 (SD 4070) nmol/1 in the first week, 5580 (SD 6590) nmol/l in the second week, 3120 (SD 1950) nmol/1 in the third week and 2430 (SD 1200) nmol/l in the fourth week). Romain et al. ${ }^{(10)}$ mentioned that the highest values of total polyamines were reached at the end of the first week of lactation and, in agreement with the present results, they reported the same decreasing trend at the end of the first month of lactation.

We report for the first time the polyamine content in preterm human milk samples. The polyamine concentration was significantly higher in preterm than in term milk samples during the first month of lactation. The higher levels of polyamines in preterm milk could reflect the higher protein content reported in preterm milk than in term milk. Sanguansermsri et $a l{ }^{(8)}$ reported that the protein content of milk samples analysed matched the putrescine and cadaverine contents. In fact, preterm milk has been seen to contain significantly higher concentrations of protein, $\mathrm{Na}$ and $\mathrm{Cl}$, and lower concentrations of lactose ${ }^{(18)}$. The mean concentrations of protein, $\mathrm{Na}, \mathrm{Cl}$ and $\mathrm{K}$ in early preterm milk are sufficient 
to meet the estimated requirements for the preterm infant ${ }^{(18)}$. The relationship between the DNA and RNA, and the spermine and spermidine concentrations reported in both human and cows' milk during early lactation ${ }^{(8)}$ suggest that an important role of polyamines is the synthesis of nucleic acids and proteins ${ }^{(8,19,20)}$.

Some authors showed that intestinal permeability to macromolecules differs in premature babies when they are fed breast milk compared with those fed infant formulas ( $\mathrm{J}$ Senterre, J Rigo, G Forget, G Dandrifosse and N Romain, unpublished results). This difference does not seem to be present when powdered milk is supplemented with polyamines at the concentration found in breast milk; spermine increases the proliferation and differentiation of lymphocytes isolated from the tonsils of children ${ }^{(21)}$. The increased maturation of the small intestine leads to gut closure, which might decrease the transfer of antigens from the lumen to the blood.

Romain et al. ${ }^{(10)}$ proposed that the putative protective effect of human milk against allergies, compared with classical infant formulas, could, at least partially, be explained by its polyamine (spermine, spermidine and putrescine) concentration and the role of these compounds in the postnatal maturation of the small intestine. In mice, infant formula supplemented with polyamines alters the intestinal microbiota, which could interfere with the immune response of animals ${ }^{(22,23)}$. Mean concentrations of spermidine/spermine in milk drunk during the first postnatal month by allergic children and nonallergic children were significantly different $(P=0.003$ and $P<0 \cdot 001)^{(10)}$. The spermidine/spermine ratio was significantly higher in the transition milk of term infants than in that for preterm infants; in contrast, in mature milk the spermidine/ spermine ratio value was significantly higher in the milk produced for preterm infants than term infants ${ }^{(10)}$. Other authors have demonstrated that the spermidine/spermine ratio in the lactating mammary gland of rats is higher than that during pregnancy. Generally, this enhancement of the spermidine/ spermine ratio initially signals increased biosynthetic activity of a tissue and, more specifically, signals an increased biosynthetic capacity for RNA and protein ${ }^{(8)}$. Maybe the discrepancy in this ratio between preterm and term milk samples was due to the different requirements of such babies because of the differences in gestational age. In fact, milk formulas for preterm babies sometimes require protein supplementation because of the special requirements of such babies.

Preterm human milk is theoretically more suitable for the premature infant than either mature or term human milk, which might be deficient in specific nutrients for the very low birth weight baby ${ }^{(24)}$. Other key nutrients such as longchain PUFA, arachidonic acid and DHA, as well as essential fatty acids, are also found in higher concentrations in early human milk samples of mothers giving birth to very low birth weight preterm infants than is the case for full-term infants ${ }^{(25)}$. In agreement with these authors, the practical implications of the present results highlight the importance of providing their own mother's milk to preterm infants. If it is necessary to use banked term human milk, it might be of interest to fortify it with polyamines, although this would require suitable clinical trials with premature infants to demonstrate possible benefits.

In conclusion, the present results indicate that the human milk of preterm babies has a significantly higher content of polyamines than term human milk and a different spermidine/spermine ratio, both of which could be important for the gut maturation of neonates and for designing infant formulas for premature babies.

\section{Acknowledgements}

J. P.-Z. and M. S.-M. contributed to the analysis, data interpretation and manuscript preparation. J. M. N. and V. B. and S. Z. contributed to the data collection and manuscript preparation. M. R.-P. and M. R. carried out data interpretation and manuscript preparation. E. L. contributed to the study design, data interpretation and manuscript preparation. All authors have read and approved the final manuscript. The present work was supported by the company Ordesa, S.L. (Spain). M. R.-P. and M. R. are employees of Ordesa, S.L. None of the authors has any conflicts of interest.

\section{References}

1. Pegg AE \& McCann PP (1982) Polyamine metabolism and function. Am J Physiol 243, C212-C221.

2. Seiler N \& Heby O (1988) Regulation of cellular polyamines in mammals. Acta Biochim Biophys Hung 23, 1-36.

3. McCormack SA \& Johnson LR (1991) Role of polyamines in gastrointestinal mucosal growth. Am J Physiol 260, G795-G806.

4. Larqué E, Sabater-Molina M \& Zamora S (2007) Biological significance of dietary polyamines. Nutrition 23, 87-95.

5. Bardocz S, Duguid TJ, Brown DS, et al. (1995) The importance of dietary polyamines in cell regeneration and growth. BrJ Nutr 73, 819-828.

6. Milovic V (2001) Polyamines in the gut lumen: bioavailability and biodistribution. Eur $J$ Gastroenterol Hepatol $\mathbf{1 3}$ 1021-1025.

7. Sabater-Molina M, Larqué E, Torrella F, et al. (2009) Effects of dietary polyamines at physiologic doses in early-weaned piglets. Nutrition 25, 940-946.

8. Sanguansermsri J, Gyorgy P \& Zilliken F (1974) Polyamines in human and cow's milk. Am J Clin Nutr 27, 859-865.

9. Pollack PF, Koldovsky O \& Nishioka K (1992) Polyamines in human and rat milk and infant formulas. Am J Clin Nutr $\mathbf{5 6}$ $371-375$.

10. Romain N, Dandrifosse G, Jeusette F, et al. (1992) Polyamine concentration in rat milk and food, human milk, and infant formulas. Pediatr Res 32, 58-63.

11. Löser C (2000) Polyamines in human and animal milk. Br J Nutr 84, S55-S58.

12. Dorhout B, Van Beusekom CM, Huisman M, et al. (1996) Estimation of twenty-four hour polyamine intake from mature human milk. J Pediatr Gastroenterol Nutr 23, 298-302.

13. Buts JP (1996) Polyamines in milk. Ann Nestle 54, 98-104.

14. Buts JP, Keyser N, Raedemaeker L, et al. (1995) Polyamine profiles in human milk, infant artificial formulas, and semielemental diets. J Pediatr Gastroenterol Nutr 21, 44-49.

15. Peulen O, Dewé W, Dandrifosse G, et al. (1998) The relationship between spermine content of human milk 
during the first postnatal month and allergy in children. Public Health Nutr 1, 181-184.

16. Motyl T, Ploszaj T, Wojtasik A, et al. (1995) Polyamines in cow's and sow's milk. Comp Biochem Physiol B: Biochem Mol Biol 111, 427-433.

17. Duchén K \& Thorell L (1999) Nucleotide and polyamine levels in colostrum and mature milk in relation to maternal atopy and atopic development in the children. Acta Paediatr 88, $1338-1343$.

18. Gross SJ, Geller J \& Tomarelli RM (1981) Composition of breast milk from mothers of preterm infants. Pediatrics $\mathbf{6 8}$, 490-493.

19. Moruzzi G, Barbiroli B \& Caldarera CM (1968) Polyamines and nucleic acid metabolism in chick embryo. Incorporation of labelled precursors into nucleic acids of subcellular fractions and polyribosomal patterns. Biochem J 107, 609-613.

20. Russell D \& Snyder SH (1968) Amine synthesis in rapidly growing tissues: ornithine decarboxylase activity in regenerating rat liver, chick embryo, and various tumors. Proc Natl Acad Sci US A 60, 1420-1427.

21. Dandrifosse G, Peulen O, El Khefif N, et al. (2000) Are milk polyamines preventive agents against food allergy? Proc Nutr Soc 9, 81-86.

22. Pérez-Cano FJ, González-Castro A, Castellote C, et al. (2010) Influence of breast milk polyamines on suckling rat immune system maturation. Dev Comp Immunol 34, 210-218.

23. Gómez-Gallego C, Collado MC, Ilo T, et al. (2012) Infant formula supplemented with polyamines alters the intestinal microbiota in neonatal BALB/cOlaHsd mice. J Nutr Biochem 23, 1508-1513.

24. Lemons JA, Moye L, Hall D, et al. (1982) Differences in the composition of preterm and term human milk during early lactation. Pediatr Res 16, 113-117.

25. Kovács A, Funke S, Marosvölgyi T, et al. (2005) Fatty acids in early human milk after preterm and full-term delivery. J Pediatr Gastroenterol Nutr 41, 454-459. 